\title{
A Modified FNN Fault Diagnosis on PCVD Microwave System
}

\author{
Zhenyu Li and Hongsheng Li \\ Wuhan University of Technology, Wuhan 430070, China \\ Correspondence should be addressed to Zhenyu Li; lizhenyu_equ@foxmail.com
}

Received 29 June 2015; Accepted 13 September 2015

Academic Editor: Katsuhiro Honda

Copyright (C) $2015 \mathrm{Z}$. Li and H. Li. This is an open access article distributed under the Creative Commons Attribution License, which permits unrestricted use, distribution, and reproduction in any medium, provided the original work is properly cited.

\begin{abstract}
A modified FNN fault diagnosis algorithm is presented in this paper for microwave subsystem of Plasma Chemical Vapor Deposition (PCVD). The symptom variables are selected as the crisp inputs, and the corresponding membership functions are obtained from premeasured data as well as experts' diagnostic experience/knowledge. The prior probability and the restriction coefficients are combined into the FNN algorithm via matrix operator. This modified FNN algorithm is verified for PCVD fault diagnosis application and realizes the MIMO for multifault mode diagnosis.
\end{abstract}

\section{Introduction}

In PCVD equipment (Figure 1) which is used for making optical fiber core rod $[1,2]$, the plasma is ignited in a substrate tube through a resonator by microwave power.

The microwave power from the microwave generator is guided through the wave-guide into the resonator, generating microwave resonance in the cavity of the resonator to ignite the plasma inside the substrate quartz tube. And in the substrate tube, $\mathrm{SiCl}_{4} / \mathrm{GeCl}_{4}$ vapor and $\mathrm{O}_{2}$ are mixed and interreact in the plasma. $\mathrm{SiO}_{2}$ and $\mathrm{GeO}_{2}$, the resultant in the plasma reaction, are deposited on the inner surface of the tube.

PCVD consists of some subsystems, which perform the complicated chemical and physical reaction, including microwave and plasma, vaporizer and gas supplier, furnace, transmission, reaction piping, electrical parts, and control. Within the production, the fault detection and diagnosis on these subsystems are very important and necessary. In this paper, a fault diagnosis algorithm is proposed for PCVD microwave and plasma system.

\section{FNN for Fault Diagnosis}

In fault diagnosis of complicated system, characteristics and reasons of faults are indefinite; consequently, fuzzy algorithm is better to be used for this kind of application. FNN (Fuzzy Neural Network) is an algorithm combining fuzzy and neural networks and implements fuzzy logical inference via neural network, in which sets of input and output are specified. Sugeno Model is usually used as inference model with 5 layers [3].

Layer 1 is fuzzification layer, to produce membership grades of the crisp inputs $(X, Y)$ which belong to each of the fuzzy sets by using the membership functions. Neurons in the layer output results to the neurons in the next layer.

Layer 2 is the rule layer. Each of the neurons outputs the firing strength $W_{i}$ according to the definition of $\Pi$, such as maximum operator or product operator.

Layer 3 is the normalization layer. This layer receives inputs from all neurons in the rule layer and calculates the normalized firing strength of a given rule:

$$
\bar{W}_{i}=\frac{W_{i}}{\sum_{j=1}^{N} W_{j}} .
$$

Layer 4 is node function layer. For the 2-input node shown in Figure 2, the first-order function is

$$
z=a x+b y+c .
$$

Output of the layer is

$$
O=\bar{W} \cdot z=\bar{W} \cdot(a x+b y+c),
$$

where $a, b$, and $c$ are consequence parameters.

Layer 5 sums all inputs as total output. 


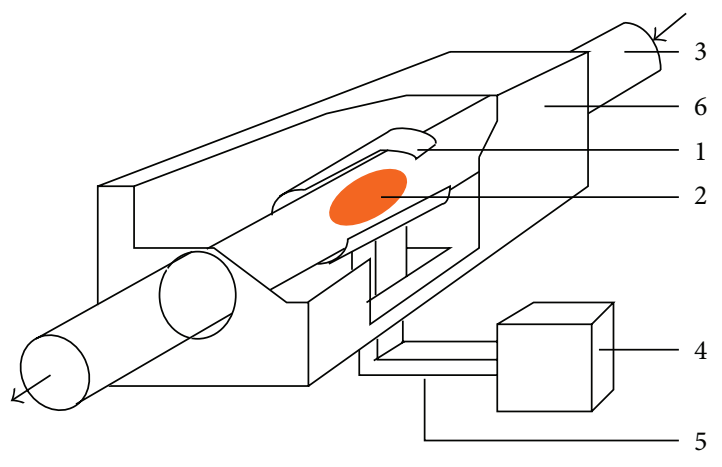
(1) Resonator
(2) Plasma
(3) Substrate tube
(4) Microwave generator
(5) Wave guide
(6) Furnace

FIGURE 1: PCVD illustration.

Some applications of fault diagnosis use FNN algorithm to predict parameters (Figure 3); then, the faults are revealed based on the difference between the measured concentration values and FNN predictions [4].

In this application, the FNN prediction is trained by a historical set of data collected during fault-free operation of the process; the process parameters are collected from online sensors. FNN is just used for parameter prediction, not for fault diagnosis directly.

In another application, the relationship between fault phenomena and fault reasons is defined with membership. Fuzzy mathematical method is used to establish diagnostic matrix; then, according to the diagnosis matrix and by BP algorithm, fault diagnosis model is generated; finally, fault reasons are output. The expert experience and historical data are both combined with membership matrix, to draw the fault reasons [5].

In a fault diagnosis application in nuclear power plant, a diagnostic solution is proposed based on the combination of FNN and data fusion (Figure 4). The method is suitable for nuclear power plant monitoring and fault diagnosis to deal with the large quantities of online measurement data. FNN is applied in local diagnosis area and data fusion is applied in global diagnosis area; they complement independent fault diagnosis by using different inputs, thereby, referring each other and generating a more reliable result [6].

\section{Modified FNN for Fault Diagnosis}

3.1. Modified FNN Algorithm. In the fault diagnosis application of the microwave system, a modified FNN is presented in this paper. By this algorithm, the probability of faults can be output directly based on a few of the real-time input signals. The FNN with Sugeno Model has obvious advantage for the nonlinear application, which involves complex computation for the identification of consequence parameters [7, 8]. Nevertheless, a new inference model in FNN Layer 4 is proposed and more suitable for this application, instead of Sugeno Model, which describes the relationship between characteristic signals and fault probability; that is, this approach is a multi-input and multioutput fault diagnosis algorithm.

The modified FNN model is shown in Figure 5.

The modification compared with FNN shown in Figure 2 is presented on Layer 4 and Layer 5.

According to Layer 4, based on the fault prior probability which is from experimental data as well as experts' diagnostic experience/knowledge to distinguish the actual fault from various external static factors [9], the probability of every fault mode is output.

According to Layer 5, outputs from Layer 4 are input into this layer, which makes the fault decision with the restriction of constraint matrix, by maximum rule.

3.2. Algorithm Realization. The following presents the modified FNN fault diagnosis algorithm.

The crisp inputs are classified into different fuzzy sets with specified membership functions.

Suppose

$$
\begin{aligned}
& x_{1}=\frac{P_{\mathrm{ref}}}{P} ; \\
& x_{2}=P_{\mathrm{ref}},
\end{aligned}
$$

where $P$ is forward power of microwave; $P_{\text {ref }}$ is reflective power of microwave.

Membership functions are defined by statistical probability of fault:

$$
\begin{aligned}
& P\left(x_{1}\right) \\
& = \begin{cases}0 & x_{1}<a_{1} \\
\frac{2}{\left(a_{1}-b_{1}\right)^{3}}\left(x_{1}-a_{1}\right)^{2}\left(x_{1}-\frac{3 b_{1}-a_{1}}{2}\right) & a_{1} \leq x_{1}<b_{1} \\
1 & b_{1} \leq x_{1} .\end{cases}
\end{aligned}
$$

Actually, $a_{1}=5 \%, b_{1}=15 \%$, and

$$
\begin{aligned}
& \mu A_{i}\left(x_{1}\right)=\left\{\begin{array}{ll}
0 & x_{1}<a_{i} \\
\frac{x_{1}-a_{i}}{b_{i}-a_{i}} & a_{i} \leq x_{1}<b_{i} \\
\frac{c_{i}-x_{1}}{c_{i}-b_{i}} & b_{i} \leq x_{1}<c_{i} \\
0 & c_{i} \leq x_{1}
\end{array} \quad i=1,2\right. \\
& \mu A_{3}\left(x_{1}\right)= \begin{cases}0 & x_{1}<a_{3} \\
\frac{x_{1}-a_{i}}{b_{i}-a_{i}} & a_{3} \leq x_{1}<b_{3} \\
1 & b_{3} \leq x_{1} .\end{cases}
\end{aligned}
$$

Membership function of fault detected by $P_{\text {ref }} / P$ is shown as in Figure 6(b), which is described by (5b) and (5c). 


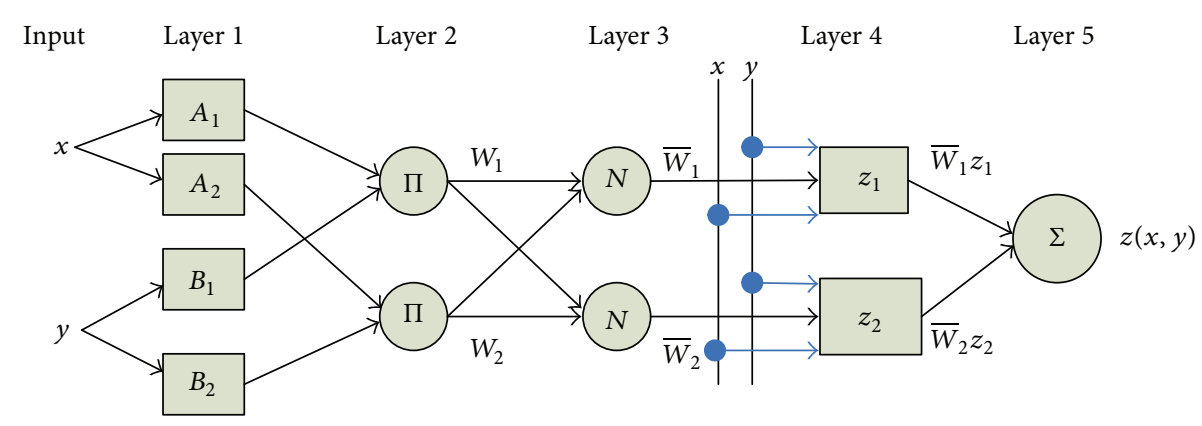

Figure 2: FNN with Sugeno Model.

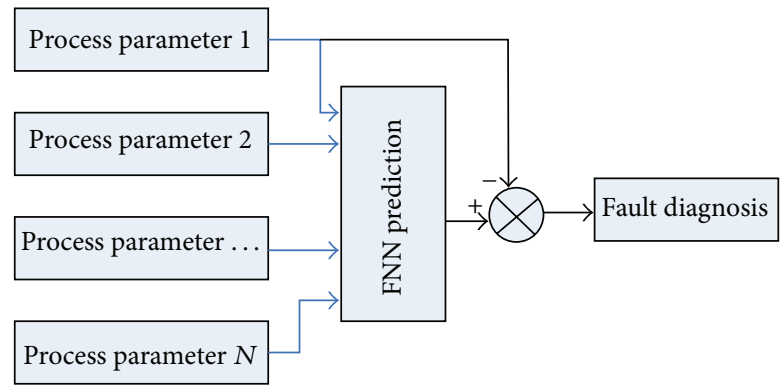

FIGURE 3: FNN prediction for fault diagnosis.

The membership function is based on the statistical probability of fault (Figure 6(a)):

$$
P\left(x_{2}\right)= \begin{cases}0 & x_{2}<a_{2} \\ \frac{x_{2}-a_{2}}{b_{2}-a_{2}} & a_{2} \leq x_{2}<b_{2} \\ 1 & b_{2} \leq x_{2} .\end{cases}
$$

In production, $a_{2}=300, b_{2}=1000$ (unit: Watt), and

$$
\begin{aligned}
& \mu B_{1}\left(x_{2}\right)= \begin{cases}1 & x_{2}<300 \\
\frac{1000-x_{2}}{700} & 300 \leq x_{2}<1000 \\
0 & 1000 \leq x_{2},\end{cases} \\
& \mu B_{2}\left(x_{2}\right)= \begin{cases}0 & x_{2}<300 \\
\frac{x_{2}-300}{700} & 300 \leq x_{2}<1000 \\
1 & 1000 \leq x_{2} .\end{cases}
\end{aligned}
$$

Membership function of fault detected by $P_{\text {ref }}$ is shown as in Figure 7(b), which is described by (6b) and (6c). The membership function is based on the statistical probability of fault (Figure 7(a)).

The realization of the fault diagnosis (Figure 5) is illustrated as below.

In production, microwave mismatch is a common fault in the microwave system; the phenomena appear as the measurement value of $P_{\text {ref }}$ or/and $P_{\text {ref }} / P$ higher than normal.

The mismatch fault is divided into 4 fault modes (Figure 8), wave-guide alignment problem, substrate-tube bend, autotuner fault, and magnetron fault. The fault weight $w$ is calculated from statistic data.

$w_{i, 1}, w_{i, 2}$, and $w_{i, 3}$ are corresponding to different fuzzy rule combination; for example, $w_{i, 1}$ is corresponding to Rule $A_{1}+$ $B_{1}$ (Figure 5).

Based on the above data of weight, the algorithm of Layer 4 is able to design as below:

$$
\mathbf{z}_{i}=\left(\begin{array}{c}
\mu_{F_{1}, i} \\
\mu_{F_{2}, i} \\
\mu_{F_{3}, i} \\
\mu_{F_{4}, i}
\end{array}\right)=\overline{w_{i}} \cdot\left(\begin{array}{c}
w_{1, i} \\
w_{2, i} \\
w_{3, i} \\
w_{4, i}
\end{array}\right) \quad i=1,2,3 .
$$

Decision algorithm of Layer 5 is designed as below:

$$
\mathbf{z}=\left(\begin{array}{l}
\mu_{F_{1}} \\
\mu_{F_{2}} \\
\mu_{F_{3}} \\
\mu_{F_{4}}
\end{array}\right)=\sum_{i=1}^{3} Z_{i} \cdot\left(\begin{array}{cccc}
r_{1,1} & r_{1,2} & r_{1,3} & r_{1,4} \\
r_{2,1} & r_{2,2} & r_{2,3} & r_{2,4} \\
r_{3,1} & r_{3,2} & r_{3,3} & r_{3,4} \\
r_{4,1} & r_{4,2} & r_{4,3} & r_{4,4}
\end{array}\right)
$$

where constraint matrix $\mathbf{r}$ is determined by physical condition of fault mode, including intrinsic restriction and dependent restriction, and $r_{i, j}(i=j)$ is intrinsic restriction coefficient to judge the probability of fault mode $F_{i}$, determined by the factors related to the fault mode directly; and $r_{i, j}(i \neq j)$ is dependent restriction coefficient to judge the probability of fault mode $F_{i}$, determined by the factors related to the fault mode indirectly.

For example, the intrinsic restriction coefficient is a function to describe the working status of the specified part in the equipment. As for the wave guide, its alignment is not qualified absolutely after reinstallation; after confirmation by running, it keeps stable for a certain period; then, after the stable period, the alignment will turn to abnormal status caused by movement vibration and structure deformation. So we define the function of intrinsic restriction coefficient in Figure 9.

The intrinsic restriction coefficient of fault mode $F_{1}$ (wave-guide alignment) is $r_{1,1}=1$, if the wave guide was reinstalled before running, and $r_{1,1}=0.1$, if the wave guide was not changed during 60 days (Figure 9).

Fault mode $F_{4}$ (magnetron fault) $r_{4,4}$ is dependent on the reliability function of magnetron life time (Figure 10 and (9)). 


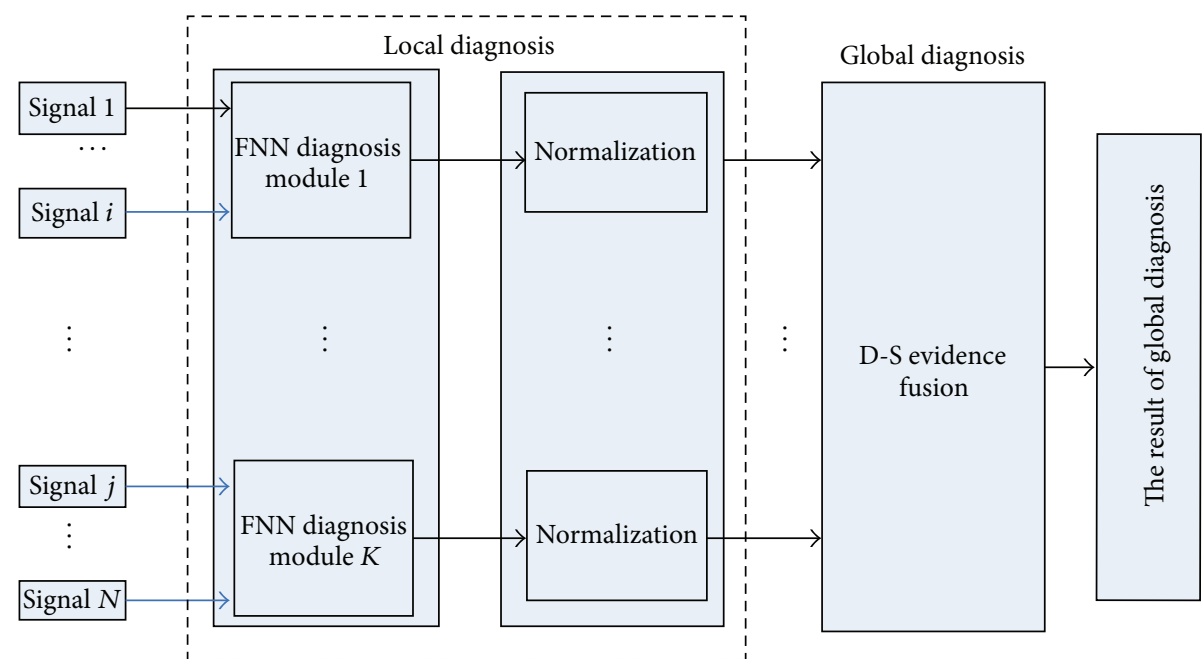

FIGURE 4: The integration of FNN and data fusion.
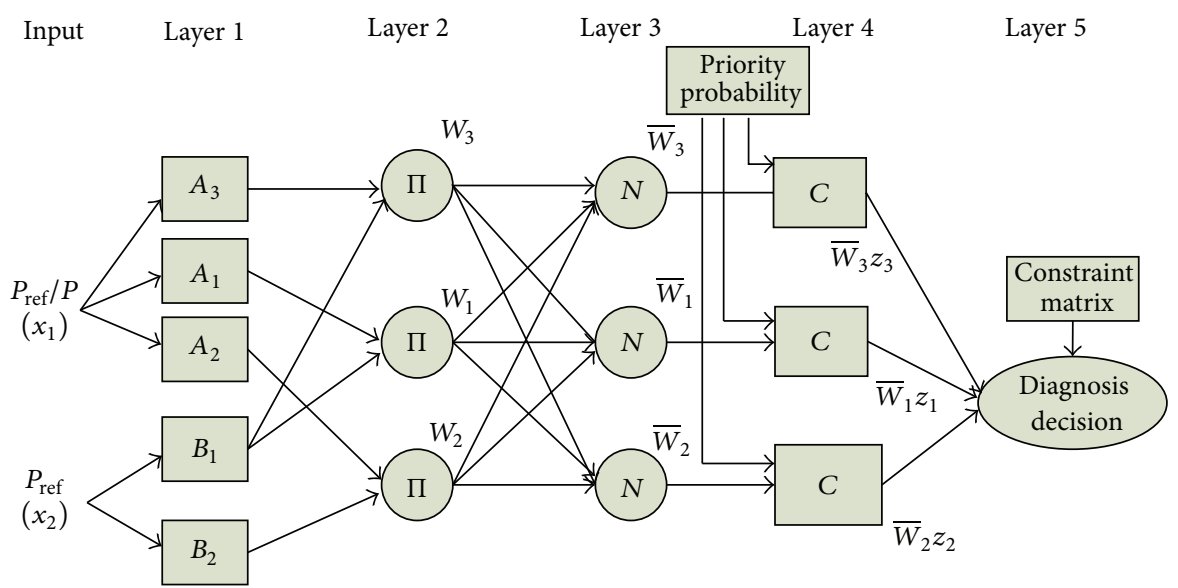

FIgURE 5: Modified FNN fault diagnosis where $\Pi$ is maximum operator.

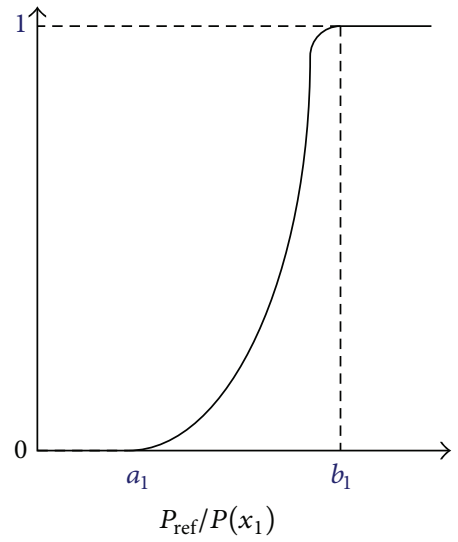

(a)

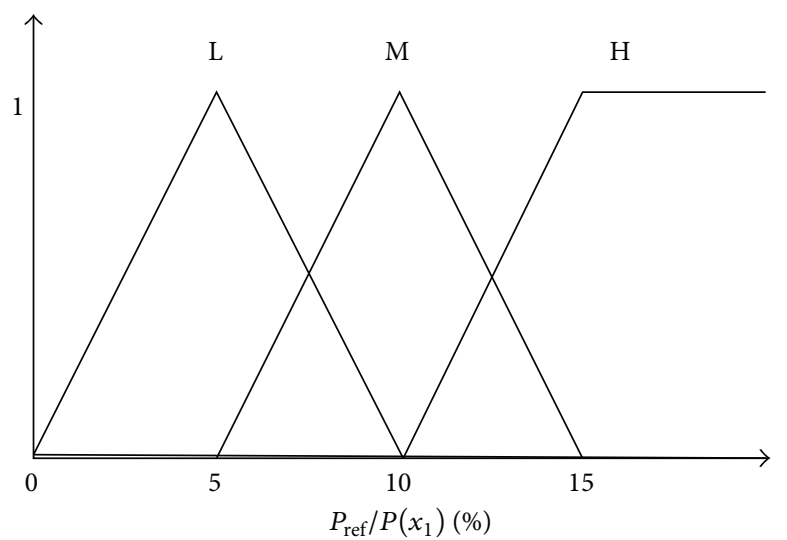

(b)

Figure 6: (a) Fault probability by $P_{\text {ref }} / P$. (b) Membership function of fault by $P_{\text {ref }} / P$. 


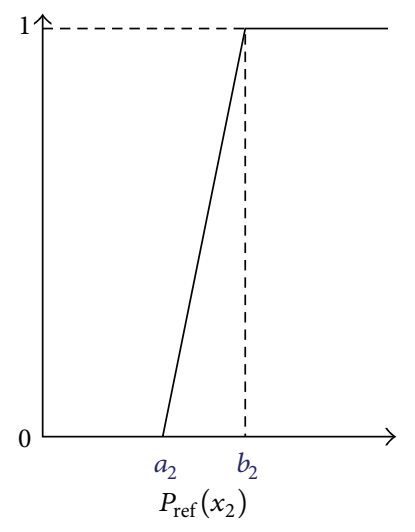

(a)

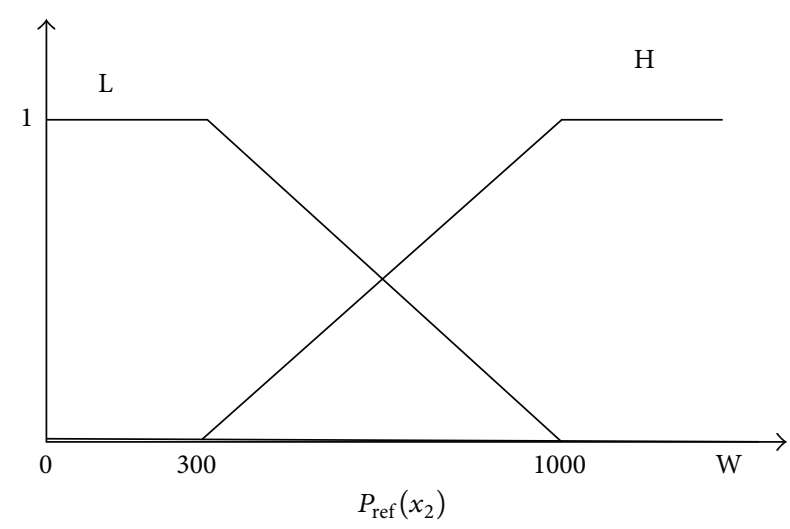

(b)

FIgURE 7: (a) Fault probability by $P_{\text {ref }}$ (b) Membership function of fault by $P_{\text {ref }}$.

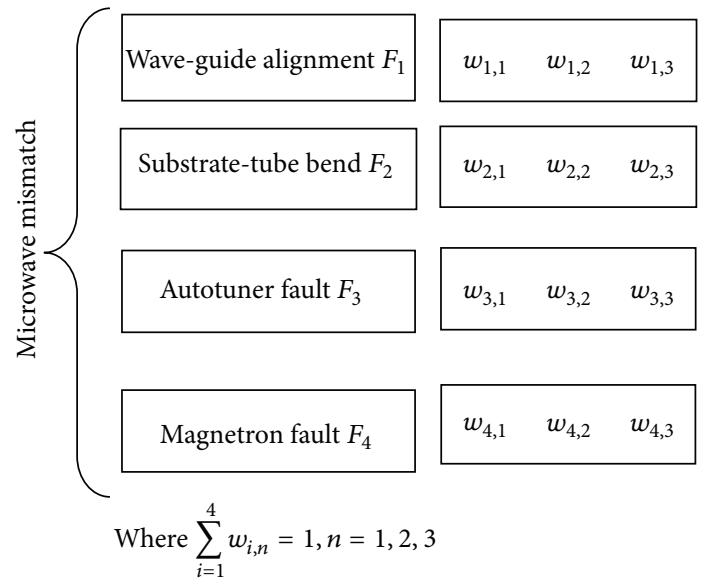

FIGURE 8: Weight of fault.

Normally, the life time of magnetron is about 5000 hours. According to the function, the fault probability is very low if the usage time of magnetron is less than 4000 hours; that is, $r_{4,4}=0.1$ :

$$
R(t)= \begin{cases}0.1 & t<a \\ \frac{2}{(a-b)^{3}}(t-a)^{2}\left(t-\frac{3 b-a}{2}\right) & a \leq t<b \\ 1 & b \leq t .\end{cases}
$$

Actually, $a=4000$ and $b=6000$ (unit: hour).

Dependent restriction coefficient represents the associative influence factors from other faults. For example, the magnetron fault could be caused by the autotuner fault based on the theory of microwave application. In case the moding fault generated by autotuner fault appears in the microwave system, the filament in magnetron would be damaged. That means fault modes $F_{3}$ (autotuner fault) and $F_{4}$ (magnetron fault) are associative and $F_{3}$ affects $F_{4}$; then, $r_{4,3}>0$, that is, to increase the weight of $F_{4}$ by $F_{3}$. Conversely, since there is no associative fault mode to affect $F_{1}$, the dependent restriction coefficients of $F_{1}$ are zero totally.

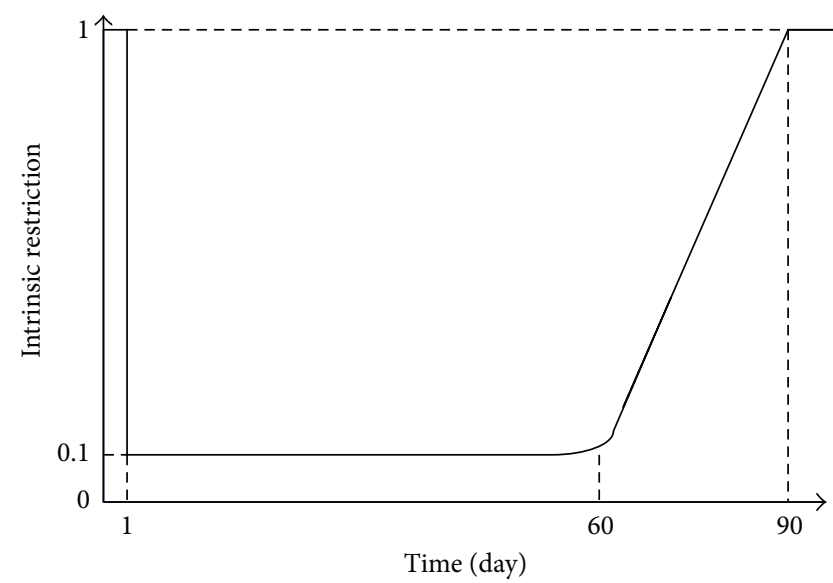

FIGURE 9: Intrinsic restriction coefficient function of wave-guide alignment.

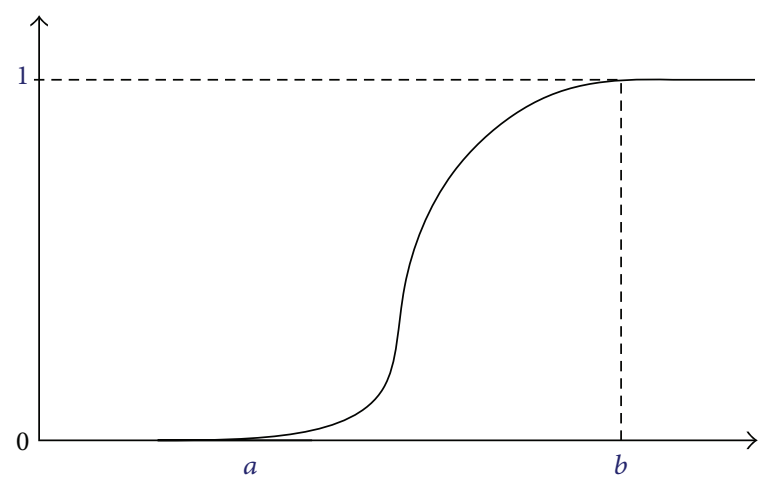

FIgURE 10: Reliability function.

3.3. Production Instance. The fault diagnosis algorithm is illustrated as below.

Crisp inputs are

$$
\begin{aligned}
& x_{1}=9 \%, \\
& x_{2}=500 .
\end{aligned}
$$


According to (5b) and (5c),

$$
\begin{aligned}
& \mu A_{1}\left(x_{1}\right)=0.20, \\
& \mu A_{2}\left(x_{1}\right)=0.80, \\
& \mu A_{3}\left(x_{1}\right)=0 .
\end{aligned}
$$

According to (6b) and (6c),

$$
\begin{aligned}
& \mu B_{1}\left(x_{2}\right)=0.60, \\
& \mu B_{2}\left(x_{2}\right)=0.30 .
\end{aligned}
$$

The prior probability is

$$
\begin{aligned}
\left(\begin{array}{lll}
w_{1,1} & w_{1,2} & w_{1,3} \\
w_{2,1} & w_{2,2} & w_{2,3} \\
w_{3,1} & w_{3,2} & w_{3,3} \\
w_{4,1} & w_{4,2} & w_{4,3}
\end{array}\right) & =\left(\begin{array}{lll}
0.40 & 0.25 & 0.15 \\
0.30 & 0.30 & 0.25 \\
0.15 & 0.15 & 0.25 \\
0.15 & 0.30 & 0.35
\end{array}\right), \\
\mathbf{z}_{1} & =\left(\begin{array}{c}
0.16 \\
0.12 \\
0.06 \\
0.06_{i}
\end{array}\right), \\
\mathbf{z}_{2} & =\left(\begin{array}{c}
0.15 \\
0.18 \\
0.09 \\
0.18_{i}
\end{array}\right), \\
\mathbf{z}_{3} & =\left(\begin{array}{c}
0 \\
0 \\
0 \\
0
\end{array}\right) .
\end{aligned}
$$

And the other conditions are as follows:

The resonator is replaced recently; the magnetron has been used for 4500 hours.

Dependent restriction coefficient $\left(F_{3}\right.$ to $\left.F_{4}\right) r_{4,3}$ is set to 0.4 :

$$
r=\left(\begin{array}{cccc}
1 & 0 & 0 & 0 \\
0 & 1 & 0 & 0 \\
0 & 0 & 1 & 0 \\
0 & 0 & 0.4 & 0.5
\end{array}\right)
$$

So,

$$
\mathbf{z}=\left(\begin{array}{c}
0.31 \\
0.30 \\
0.15 \\
0.24
\end{array}\right)\left(\begin{array}{cccc}
1 & 0 & 0 & 0 \\
0 & 1 & 0 & 0 \\
0 & 0 & 1 & 0 \\
0 & 0 & 0.4 & 0.5
\end{array}\right)=\left(\begin{array}{l}
0.31 \\
0.30 \\
0.15 \\
0.18
\end{array}\right) .
$$

The results of FNN fault diagnosis of 60 sets of historical data are listed in Table 1.

Where the matrix of prior probability is shown as (13), the matrix of restriction coefficient is shown as below.

When usage time of magnetron is in the duration of 0 to 4000 hours,

$$
r=\left(\begin{array}{cccc}
1 & 0 & 0 & 0 \\
0 & 0.15 & 0 & 0 \\
0 & 0 & 0.1 & 0 \\
0 & 0 & 0 & 0.1
\end{array}\right)
$$

When usage time of magnetron is about 5000 hours,

$$
r=\left(\begin{array}{cccc}
1 & 0 & 0 & 0 \\
0 & 0.15 & 0 & 0 \\
0 & 0 & 0.1 & 0 \\
0 & 0 & 0.6 & 0.5
\end{array}\right)
$$

When usage time of magnetron is about 6000 hours,

$$
r=\left(\begin{array}{cccc}
1 & 0 & 0 & 0 \\
0 & 0.15 & 0 & 0 \\
0 & 0 & 0.1 & 0 \\
0 & 0 & 0.5 & 1
\end{array}\right)
$$

In particular, in some circumstances, the fault diagnosis algorithm is still effective. For example, under this kind of circumstance: the wave guide has kept no change for 10 to 20 days, the substrate tube is qualified, the autotuner is of normal status, the usage time of magnetron is in the duration of 0 to 4000 hours, and it seems that every part is staying in normal status, but actually the faults still appear as the alignment turning worse by vibration and moving, or the substrate-tube deforming by heating or stress. Table 2 shows the diagnosis results by the algorithm.

As the fault decision is by maximum rule, the results of the diagnosis match actual faults very well, with high diagnostic accuracy (Table 2). Of course, if the calculation results of faults probability are approximate, the diagnosis decision is hard to make correctly. So the new input should be introduced into the diagnosis algorithm, in order to improve the accuracy.

3.4. Comparison with Information Fusion. Next, we will compare the diagnosis result of Information Fusion Diagnosis with the modified FNN diagnosis.

In the Information Fusion Diagnosis, Dempster-Shafer evidence combination rules with strong classification capability for faults of evidences data overlapping are applied to obtain Information Fusion results [10]. First, the data (evidences) from sensing sources are pretreated, and then the basic confidence degree is combined by D-S rules; finally, the fusion results are selected according to specified decision rule (Figure 11). 
TABLE 1: The results of FNN fault diagnosis.

\begin{tabular}{|c|c|c|c|c|c|c|c|c|c|c|c|}
\hline \multirow{2}{*}{ Time (hr) } & \multicolumn{3}{|c|}{ Fault symptom } & \multicolumn{4}{|c|}{ Fault mode probability } & \multicolumn{4}{|c|}{ Actual fault } \\
\hline & I.R.C. & $P_{\text {ref }} / P$ & $P_{\text {ref }}$ & $F_{1}$ & $F_{2}$ & $F_{3}$ & $F_{4}$ & $F_{1}$ & $F_{2}$ & $F_{3}$ & $F_{4}$ \\
\hline \multirow[t]{20}{*}{$0 \sim 4000$} & 0.1 & 0.16 & 1000 & 0.6809 & 0.1404 & 0.0681 & 0.1106 & $\mathrm{~T}$ & \multirow{20}{*}{$\mathrm{X}$} & & \\
\hline & 0.1 & 0.13 & 800 & 0.7268 & 0.1279 & 0.0569 & 0.0884 & $\mathrm{~T}$ & & & \\
\hline & 0.1 & 0.10 & 600 & 0.7495 & 0.1224 & 0.0503 & 0.0778 & $\mathrm{~T}$ & & & \\
\hline & 0.1 & 0.07 & 400 & 0.7566 & 0.1174 & 0.0533 & 0.0726 & $\mathrm{~T}$ & & & \\
\hline & 0.1 & 0.20 & 1000 & 0.6809 & 0.1404 & 0.0681 & 0.1106 & $\mathrm{~T}$ & & & \\
\hline & 0.1 & 0.16 & 800 & 0.7077 & 0.1317 & 0.0638 & 0.0968 & $\mathrm{~T}$ & & & \\
\hline & 0.1 & 0.12 & 600 & 0.7526 & 0.1202 & 0.0516 & 0.0755 & & & & \\
\hline & 0.1 & 0.08 & 400 & 0.7548 & 0.1187 & 0.0526 & 0.0739 & $\mathrm{~T}$ & & & \\
\hline & 0.1 & 0.25 & 1000 & 0.6809 & 0.1404 & 0.0681 & 0.1106 & $\mathrm{~T}$ & & & \\
\hline & 0.1 & 0.20 & 800 & 0.7077 & 0.1317 & 0.0638 & 0.0968 & $\mathrm{~T}$ & & & \\
\hline & 0.1 & 0.15 & 600 & 0.7307 & 0.1242 & 0.0602 & 0.0850 & & & $\mathrm{X}$ & \\
\hline & 0.1 & 0.10 & 400 & 0.7521 & 0.1206 & 0.0514 & 0.0759 & $\mathrm{~T}$ & & & \\
\hline & 0.1 & 0.33 & 1000 & 0.6809 & 0.1404 & 0.0681 & 0.1106 & $\mathrm{~T}$ & & & \\
\hline & 0.1 & 0.27 & 800 & 0.7077 & 0.1317 & 0.0638 & 0.0968 & $\mathrm{~T}$ & & & \\
\hline & 0.1 & 0.20 & 600 & 0.7307 & 0.1242 & 0.0602 & 0.0850 & & & $\mathrm{X}$ & \\
\hline & 0.1 & 0.13 & 400 & 0.7566 & 0.1174 & 0.0533 & 0.0726 & $\mathrm{~T}$ & & & \\
\hline & 0.1 & 0.50 & 1000 & 0.6809 & 0.1404 & 0.0681 & 0.1106 & $\mathrm{~T}$ & & & \\
\hline & 0.1 & 0.40 & 800 & 0.7077 & 0.1317 & 0.0638 & 0.0968 & $\mathrm{~T}$ & & & \\
\hline & 0.1 & 0.30 & 600 & 0.7307 & 0.1242 & 0.0602 & 0.0850 & $\mathrm{~T}$ & & & \\
\hline & 0.1 & 0.20 & 400 & 0.7518 & 0.1173 & 0.0569 & 0.0740 & $\mathrm{~T}$ & & & \\
\hline \multirow[t]{20}{*}{5000} & 0.5 & 0.16 & 1000 & 0.3678 & 0.0759 & 0.0368 & 0.5195 & $\mathrm{~T}$ & & & \\
\hline & 0.5 & 0.13 & 800 & 0.4289 & 0.0755 & 0.0335 & 0.4621 & $\mathrm{~T}$ & & & \\
\hline & 0.5 & 0.10 & 600 & 0.4647 & 0.0759 & 0.0312 & 0.4282 & $\mathrm{~T}$ & & & \\
\hline & 0.5 & 0.07 & 400 & 0.4698 & 0.0729 & 0.0331 & 0.4242 & $\mathrm{~T}$ & & & \\
\hline & 0.5 & 0.20 & 1000 & 0.3678 & 0.0759 & 0.0368 & 0.5195 & $\mathrm{~T}$ & & & \\
\hline & 0.5 & 0.16 & 800 & 0.3998 & 0.0744 & 0.0361 & 0.4897 & $\mathrm{~T}$ & & & \\
\hline & 0.5 & 0.12 & 600 & 0.4669 & 0.0746 & 0.0320 & 0.4264 & $\mathrm{~T}$ & & & \\
\hline & 0.5 & 0.08 & 400 & 0.4685 & 0.0737 & 0.0326 & 0.4252 & $\mathrm{~T}$ & & & \\
\hline & 0.5 & 0.25 & 1000 & 0.3678 & 0.0759 & 0.0368 & 0.5195 & $\mathrm{~T}$ & & & \\
\hline & 0.5 & 0.20 & 800 & 0.3998 & 0.0744 & 0.0361 & 0.4897 & $\mathrm{~T}$ & & & \\
\hline & 0.5 & 0.15 & 600 & 0.4296 & 0.0730 & 0.0354 & 0.4621 & $\mathrm{~T}$ & & & \\
\hline & 0.5 & 0.10 & 400 & 0.4665 & 0.0748 & 0.0319 & 0.4268 & $\mathrm{~T}$ & & & \\
\hline & 0.5 & 0.33 & 1000 & 0.3678 & 0.0759 & 0.0368 & 0.5195 & $\mathrm{~T}$ & & & \\
\hline & 0.5 & 0.27 & 800 & 0.3998 & 0.0744 & 0.0361 & 0.4897 & $\mathrm{~T}$ & & & \\
\hline & 0.5 & 0.20 & 600 & 0.4296 & 0.0730 & 0.0354 & 0.4621 & & & & $\mathrm{~T}$ \\
\hline & 0.5 & 0.13 & 400 & 0.4698 & 0.0729 & 0.0331 & 0.4242 & $\mathrm{~T}$ & & & \\
\hline & 0.5 & 0.50 & 1000 & 0.3678 & 0.0759 & 0.0368 & 0.5195 & & & & $\mathrm{~T}$ \\
\hline & 0.5 & 0.40 & 800 & 0.3998 & 0.0744 & 0.0361 & 0.4897 & & & & $\mathrm{~T}$ \\
\hline & 0.5 & 0.30 & 600 & 0.4296 & 0.0730 & 0.0354 & 0.4621 & & & & $\mathrm{~T}$ \\
\hline & 0.5 & 0.20 & 400 & 0.3854 & 0.0601 & 0.0291 & 0.5253 & & & & $\mathrm{~T}$ \\
\hline \multirow[t]{9}{*}{6000} & 1 & 0.16 & 1000 & 0.2914 & 0.0601 & 0.0291 & 0.6193 & & & & $\mathrm{~T}$ \\
\hline & 1 & 0.13 & 800 & 0.3494 & 0.0615 & 0.0273 & 0.5617 & & & & $\mathrm{~T}$ \\
\hline & 1 & 0.10 & 600 & 0.3840 & 0.0627 & 0.0258 & 0.5275 & & & & $\mathrm{~T}$ \\
\hline & 1 & 0.07 & 400 & 0.3940 & 0.0612 & 0.0278 & 0.5171 & & & & $\mathrm{~T}$ \\
\hline & 1 & 0.20 & 1000 & 0.2914 & 0.0601 & 0.0291 & 0.6193 & & & & $\mathrm{~T}$ \\
\hline & 1 & 0.16 & 800 & 0.3231 & 0.0601 & 0.0291 & 0.5876 & & & & $\mathrm{~T}$ \\
\hline & 1 & 0.12 & 600 & 0.3884 & 0.0620 & 0.0266 & 0.5229 & & & & $\mathrm{~T}$ \\
\hline & 1 & 0.08 & 400 & 0.3915 & 0.0616 & 0.0273 & 0.5197 & & & & $\mathrm{~T}$ \\
\hline & 1 & 0.25 & 1000 & 0.2914 & 0.0601 & 0.0291 & 0.6193 & & & & $\mathrm{~T}$ \\
\hline
\end{tabular}


TABle 1: Continued.

\begin{tabular}{|c|c|c|c|c|c|c|c|c|c|c|c|}
\hline \multirow{2}{*}{ Time (hr) } & \multicolumn{3}{|c|}{ Fault symptom } & \multicolumn{4}{|c|}{ Fault mode probability } & \multicolumn{4}{|c|}{ Actual fault } \\
\hline & I.R.C. & $P_{\text {ref }} / P$ & $P_{\text {ref }}$ & $F_{1}$ & $F_{2}$ & $F_{3}$ & $F_{4}$ & $F_{1}$ & $F_{2}$ & $F_{3}$ & $F_{4}$ \\
\hline & 1 & 0.20 & 800 & 0.3231 & 0.0601 & 0.0291 & 0.5876 & & & $\mathrm{X}$ & \\
\hline & 1 & 0.15 & 600 & 0.3537 & 0.0601 & 0.0291 & 0.5570 & & & & $\mathrm{~T}$ \\
\hline & 1 & 0.10 & 400 & 0.3876 & 0.0622 & 0.0265 & 0.5238 & & & & $\mathrm{~T}$ \\
\hline & 1 & 0.33 & 1000 & 0.2914 & 0.0601 & 0.0291 & 0.6193 & & & & $\mathrm{~T}$ \\
\hline & 1 & 0.27 & 800 & 0.3231 & 0.0601 & 0.0291 & 0.5876 & & & & $\mathrm{~T}$ \\
\hline & 1 & 0.20 & 600 & 0.3537 & 0.0601 & 0.0291 & 0.5570 & & & & $\mathrm{~T}$ \\
\hline & 1 & 0.13 & 400 & 0.3940 & 0.0612 & 0.0278 & 0.5171 & & & & $\mathrm{~T}$ \\
\hline & 1 & 0.50 & 1000 & 0.2914 & 0.0601 & 0.0291 & 0.6193 & & & & $\mathrm{~T}$ \\
\hline & 1 & 0.40 & 800 & 0.3231 & 0.0601 & 0.0291 & 0.5876 & & & & $\mathrm{~T}$ \\
\hline & 1 & 0.30 & 600 & 0.3537 & 0.0601 & 0.0291 & 0.5570 & & & & $\mathrm{~T}$ \\
\hline & 1 & 0.20 & 400 & 0.3854 & 0.0601 & 0.0291 & 0.5253 & & & & $\mathrm{~T}$ \\
\hline
\end{tabular}

I.R.C.: intrinsic restriction coefficient.

$\mathrm{T}$ : diagnosis result identical to actual fault.

$\mathrm{X}$ : actual fault.

TABLE 2: The results of FNN fault diagnosis.

\begin{tabular}{|c|c|c|c|c|c|c|c|c|c|c|}
\hline \multirow{2}{*}{ Time (hr) } & \multicolumn{2}{|c|}{ Fault symptom } & \multicolumn{4}{|c|}{ Fault mode probability } & \multicolumn{4}{|c|}{ Actual fault $\%$} \\
\hline & $P_{\text {ref }} / P$ & $P_{\text {ref }}$ & $F_{1}$ & $F_{2}$ & $F_{3}$ & $F_{4}$ & $F_{1}$ & $F_{2}$ & $F_{3}$ & $F_{4}$ \\
\hline \multirow{23}{*}{$0 \sim 4000$} & 0.16 & 1000 & 0.1758 & 0.3626 & 0.1758 & 0.2857 & 14 & 73 & 13 & 0 \\
\hline & 0.13 & 800 & 0.2101 & 0.3699 & 0.1644 & 0.2556 & 15 & 70 & 15 & 0 \\
\hline & 0.10 & 600 & 0.2303 & 0.3761 & 0.1545 & 0.2391 & 19 & 66 & 15 & 0 \\
\hline & 0.07 & 400 & 0.2371 & 0.3681 & 0.1672 & 0.2276 & 25 & 60 & 15 & 0 \\
\hline & 0.20 & 1000 & 0.1758 & 0.3626 & 0.1758 & 0.2857 & 15 & 70 & 15 & 0 \\
\hline & 0.16 & 800 & 0.1949 & 0.3626 & 0.1758 & 0.2666 & 16 & 68 & 16 & 0 \\
\hline & 0.12 & 600 & 0.2333 & 0.3726 & 0.1601 & 0.2341 & 20 & 66 & 14 & 0 \\
\hline & 0.08 & 400 & 0.2354 & 0.3701 & 0.1640 & 0.2305 & 20 & 66 & 14 & 0 \\
\hline & 0.25 & 1000 & 0.1758 & 0.3626 & 0.1758 & 0.2857 & 20 & 65 & 15 & 0 \\
\hline & 0.20 & 800 & 0.1949 & 0.3626 & 0.1758 & 0.2666 & 21 & 61 & 18 & 0 \\
\hline & 0.15 & 600 & 0.2134 & 0.3626 & 0.1758 & 0.2481 & 19 & 60 & 21 & 0 \\
\hline & 0.10 & 400 & 0.2327 & 0.3732 & 0.1590 & 0.2350 & 20 & 60 & 20 & 0 \\
\hline & 0.33 & 1000 & 0.1758 & 0.3626 & 0.1758 & 0.2857 & 23 & 56 & 21 & 0 \\
\hline & 0.27 & 800 & 0.1949 & 0.3626 & 0.1758 & 0.2666 & 25 & 55 & 20 & 0 \\
\hline & 0.20 & 600 & 0.2134 & 0.3626 & 0.1758 & 0.2481 & 25 & 56 & 19 & 0 \\
\hline & 0.13 & 400 & 0.2371 & 0.3681 & 0.1672 & 0.2276 & 25 & 54 & 21 & 0 \\
\hline & 0.50 & 1000 & 0.1758 & 0.3626 & 0.1758 & 0.2857 & 25 & 55 & 20 & 0 \\
\hline & 0.40 & 800 & 0.1949 & 0.3626 & 0.1758 & 0.2666 & 26 & 56 & 18 & 0 \\
\hline & 0.30 & 600 & 0.2134 & 0.3626 & 0.1758 & 0.2481 & 24 & 56 & 20 & 0 \\
\hline & 0.20 & 400 & 0.2325 & 0.3626 & 0.1758 & 0.2290 & 22 & 57 & 21 & 0 \\
\hline & 0.16 & 1000 & 0.1758 & 0.3626 & 0.1758 & 0.2857 & 24 & 55 & 21 & 0 \\
\hline & 0.13 & 800 & 0.2101 & 0.3699 & 0.1644 & 0.2556 & 25 & 53 & 22 & 0 \\
\hline & 0.10 & 600 & 0.2303 & 0.3761 & 0.1545 & 0.2391 & 25 & 55 & 20 & 0 \\
\hline
\end{tabular}

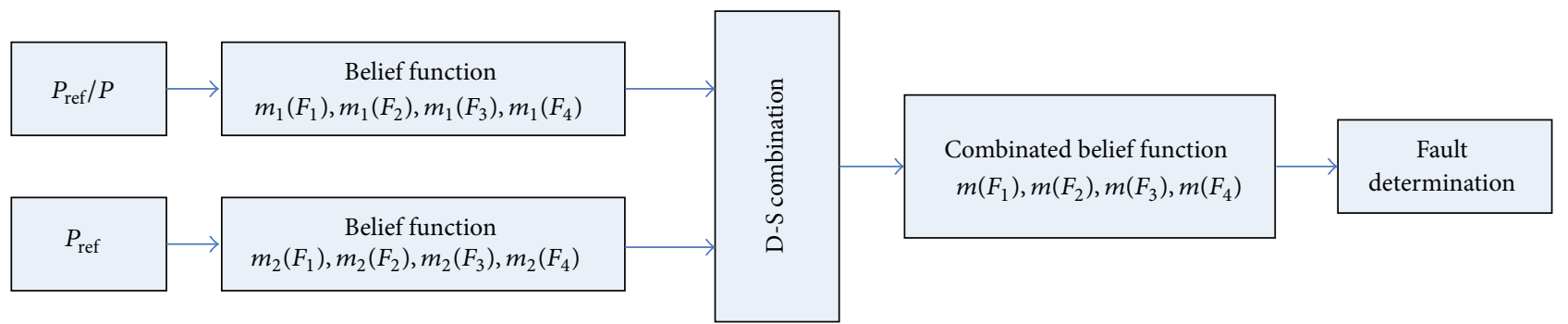

FIgURE 11: Fault diagnosis of D-S Information Fusion. 
As the limitation of the fusion algorithm, some conditions, such as magnetron usage time and wave-guide reinstallation time, cannot be introduced into the D-S Information Fusion model.

According to D-S rules, set the recognition framework

$\Theta=\{$ Wave-guide alignment, Substrate-tube bend,

Auto-tuner fault, Magnetron fault

denoted by

$$
\Theta=\left\{F_{1}, F_{2}, F_{3}, F_{4}\right\} .
$$

Corresponding evidences are $P_{\text {ref }} / P$ and $P_{\text {ref }}$.

Conventionally, evidences $\left(P_{\text {ref }} / P, P_{\text {ref }}\right)$ are analog variable, and that involves probability density $[10,11]$. This will lead to enormous calculation and lower real-time property of fault diagnosis. So, the detection signal grading in PCVD control system is used to simplify variables value, such as Normal $(\mathrm{N})$, Over High Limitation $(\mathrm{H})$, Over Higher Limitation (HH), Over Low Limitation (L), and Over Lower Limitation (LL).

Based on simplified value, the distribution of belief functions is determined via historical statistic data:

$$
\text { BLF1 }\left(\frac{P_{\text {ref }}}{P}\right)= \begin{cases}0,0,0,0 ; & \frac{P_{\text {ref }}}{P}=\mathrm{LL} \\ 0.25,0.60,0.15,0 ; & \frac{P_{\text {ref }}}{P}=\mathrm{L} \\ 0.20,0.66,0.14,0 ; & \frac{P_{\text {ref }}}{P}=\mathrm{N} \\ 0.15,0.70,0.15,0 ; & \frac{P_{\text {ref }}}{P}=\mathrm{H} \\ 0.23,0.56,0.21,0 ; & \frac{P_{\text {ref }}}{P}=\mathrm{HH}, \\ \text { BLF2 }\left(P_{\text {ref }}\right)= \begin{cases}0,0,0,0 ; & P_{\text {ref }}=\mathrm{L} \\ 0,0,0,0 ; & P_{\text {ref }}=\mathrm{L} \\ 0.20,0.60,0.20,0 ; & P_{\text {ref }}=\mathrm{N} \\ 0.24,0.56,0.20,0 ; & P_{\text {ref }}=\mathrm{H} \\ 0.20,0.65,0.15,0 ; & P_{\text {ref }}=\mathrm{HH} .\end{cases} \end{cases}
$$

Suppose $\Theta$ is a recognition framework; $m_{1}$ and $m_{2}$ are called basic belief distribution function of frame $\Theta . \Omega$ is the power set of $\Theta ; B$ and $C$ are elements of $\Omega$. D-S evidence combination result is

$$
m(A)=\frac{\sum_{B_{i} \cap C_{j}=A} m_{1}\left(B_{i}\right) m_{2}\left(C_{j}\right)}{1-k},
$$

where

$$
k=\sum_{B_{i} \cap C_{j}=\Phi} m_{1}\left(B_{i}\right) m_{2}\left(C_{j}\right) .
$$

Equation (25) is D-S evidence combination formula for 2 evidences, denoted by

$$
m(A)=m_{1}(B) \oplus m_{2}(C) .
$$

TABLE 3: Calculation of D-S Information Fusion.

\begin{tabular}{lcccc}
\hline$m$ & $F_{1}$ & $F_{2}$ & $F_{3}$ & $F_{4}$ \\
\hline$m_{1}: P_{\text {ref }} / P=\mathrm{L}$ & 0.25 & 0.60 & 0.15 & 0 \\
$m_{2}: P_{\text {ref }}=\mathrm{N}$ & 0.20 & 0.60 & 0.20 & 0 \\
\hline$m_{1} \oplus m_{2}$ & 0.1136364 & 0.818182 & 0.068182 & 0 \\
\hline$m_{1}: P_{\text {ref }} / P=\mathrm{L}$ & 0.25 & 0.60 & 0.15 & 0 \\
$m_{2}: P_{\text {ref }}=\mathrm{H}$ & 0.24 & 0.56 & 0.20 & 0 \\
\hline$m_{1} \oplus m_{2}$ & 0.1408451 & 0.788732 & 0.070423 & 0 \\
\hline$m_{1}: P_{\text {ref }} / P=\mathrm{HH}$ & 0.23 & 0.56 & 0.21 & 0 \\
$m_{2}: P_{\text {ref }}=\mathrm{HH}$ & 0.20 & 0.65 & 0.15 & 0 \\
\hline$m_{1} \oplus m_{2}$ & 0.1041903 & 0.824462 & 0.071348 & 0 \\
\hline
\end{tabular}

TABLE 4: Diagnosis result comparison of different algorithm.

\begin{tabular}{lccc}
\hline \multicolumn{2}{c}{ Samples restriction } & \multicolumn{2}{c}{ Algorithm } \\
Time (hr) & $\begin{array}{c}\text { Resonator replaced } \\
\text { recently }\end{array}$ & $\begin{array}{c}\text { Modified } \\
\text { FNN }\end{array}$ & $\begin{array}{c}\text { Information } \\
\text { Fusion }\end{array}$ \\
\hline $0 \sim 6000$ & Yes & $5.0 \%$ & $58.3 \%$ \\
$0 \sim 6000$ & No & $9.6 \%$ & $44.1 \%$ \\
$0 \sim 4000$ & No & $39.4 \%$ & $39.4 \%$ \\
\hline
\end{tabular}

So, multievidence combination formula is

$$
\begin{aligned}
m(A) & \\
& =\left[\left[\left[m_{1}(B) \oplus m_{2}(C)\right] \oplus m_{3}(D)\right] \oplus m_{4}(E)\right] \cdots,
\end{aligned}
$$

where $B, C, D, E, \ldots$ are elements of power set $\Omega$.

The below example shows D-S Information Fusion application in fault diagnosis. Since the number of evidences is 2, the evidences are set into several permutations with 2 evidences [12]. First, the belief functions are calculated according to (23) and (25), and then faults are determined by maximal value (Table 3 ).

From the result of calculation in Table 3, the distribution value of belief function for fault $F_{2}$ is obviously maximal; that is, the probability of $F_{2}$ is maximal; the distribution value of belief function for fault $F_{4}$ is zero, that is, probability zero. In this application, maximum principle is the criterion of fault determination. Obviously, the result is not identical to the actual situation. For example, on the magnetron usage time over $5000 \mathrm{hrs}$, fault $F_{1}$ and fault $F_{4}$ are possible (shown in Table 1). As the data (evidences) from sensing sources are not sufficient and the additional restriction conditions are not able to be introduced, the diagnosis results deviate from the actual ones. Of course, some correction method could be applied for the Information Fusion; that will be presented in another paper.

To demonstrate the superiority of the proposed algorithm, the simulated diagnosis results (shown as in Tables 1, 2 , and 3) are listed in Table 4 for comparison. The diagnosis results are described and evaluated by diagnosis error rate under specified fault condition.

In most circumstances, the proposed algorithm shows the superiority with much lower diagnosis error rate. Just in the specified circumstance, the two algorithms have identical results. 


\section{Conclusion}

The proposed FNN fault diagnosis algorithm combined with prior probability and restriction coefficient is approved by PCVD fault diagnosis simulation. It is a significant exploratory method where the matrix of prior probability introduced to the algorithm establishes the connection between the diagnosis result and historical diagnosis data. Another improvement for FNN algorithm is application of restriction coefficient matrix for diagnosis decision, which contains dependent restriction coefficient and intrinsic restriction coefficient, corresponding, respectively, to interaction and intrinsic property of diagnosed parts. Through the mathematic method, the characteristic and interrelationship of specified objects and historical experience are integrated into the diagnosis algorithm directly, instead of linguistic description. During the application of the diagnosis, the intrinsic restriction coefficients should be set according to actual equipment situation; and the dependent restriction coefficients and prior probability should be finely adjusted and corrected based on accumulated statistic data after a certain period.

\section{Conflict of Interests}

The authors declare that there is no conflict of interests regarding the publication of this paper.

\section{References}

[1] Z. Li and H. Li, "A new type of resonator designed for plasma chemical vapor deposition," Applied Mechanics and Materials, vol. 121-126, pp. 642-646, 2012.

[2] P. Matthijsse, D. R. Simons, S. Zhang, J. Luo, J. Vydra, and H. Fabian, "Towards the low limits of $1383 \mathrm{~nm}$ loss in PCVD enabled single mode fibre production," in Proceedings of the Optical Fiber Communication Conference (OFC '04), vol. 1, p. 3, Los Angeles, Calif, USA, February 2004.

[3] M. Saridemir, "Predicting the compressive strength of mortars containing metakaolin by artificial neural networks and fuzzy logic," Advances in Engineering Software, vol. 40, no. 9, pp. 920 927, 2009.

[4] H. Honggui, L. Ying, and Q. Junfei, "A fuzzy neural network approach for online fault detection in waste water treatment process," Computers and Electrical Engineering, vol. 40, no. 7, pp. 2216-2226, 2014.

[5] P. Zhang, R. Zhang, and X.-X. Ran, "Research of fault diagnosis in flight control system based on fuzzy neural network," Procedia Engineering, vol. 15, pp. 70-74, 2011.

[6] L. Yong-Kuo, P. Min-Jun, X. Chun-Li, and D. Ya-Xin, "Research and design of distributed fault diagnosis system in nuclear power plant," Progress in Nuclear Energy, vol. 68, pp. 97-110, 2013.

[7] S. Li and Z. Chen, "Identification of Sugeno's model," Acta Automatica Sinica, vol. 5, no. 4, pp. 58-63, 1999.

[8] M. Sugeno and T. Yasukawa, "Fuzzy-logic-based approach to qualitative modeling," IEEE Transactions on Fuzzy Systems, vol. 1, no. 1, pp. 7-31, 1993.

[9] B. D. Sekar and M. C. Dong, "Bayesian fuzzy inference nets online fault diagnosis of induction motor," in Proceedings of the 7th International Conference on Information, Communications and Signal Processing (ICICS '09), pp. 1-5, IEEE, Macau, China, December 2009.

[10] M.-A. Simard, J. Couture, and E. Bosse, "Data fusion of multiple-sensors attribute information for target-identity estimation using a Dempster-Shafer evidential combination algorithm," in Signal and Data Processing of Small Targets, vol. 2759 of Proceedings of SPIE, pp. 577-588, International Society for Optical Engineering, Washington, DC, USA, May 1996.

[11] O. Basir and X. Yuan, "Engine fault diagnosis based on multisensor information fusion using Dempster-Shafer evidence theory," Information Fusion, vol. 8, no. 4, pp. 379-386, 2007.

[12] L. Oukhellou, A. Debiolles, T. Denœux, and P. Aknin, "Fault diagnosis in railway track circuits using Dempster-Shafer classifier fusion," Engineering Applications of Artificial Intelligence, vol. 23, no. 1, pp. 117-128, 2010. 

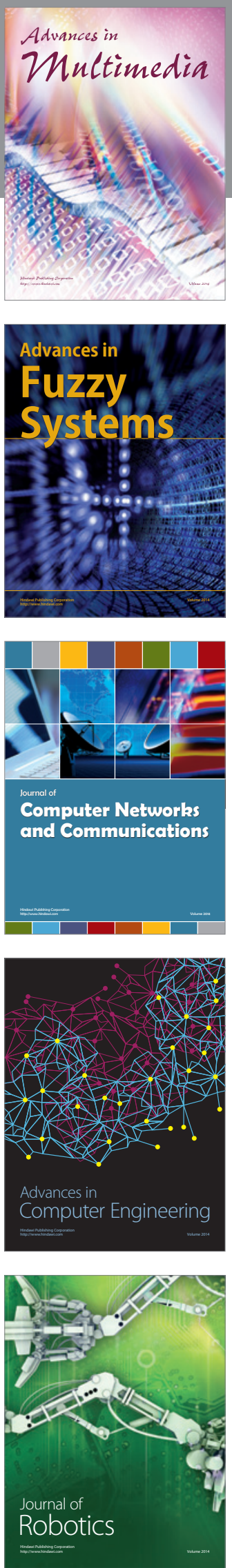

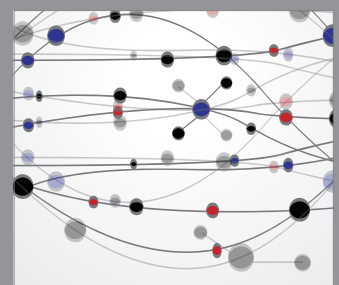

The Scientific World Journal
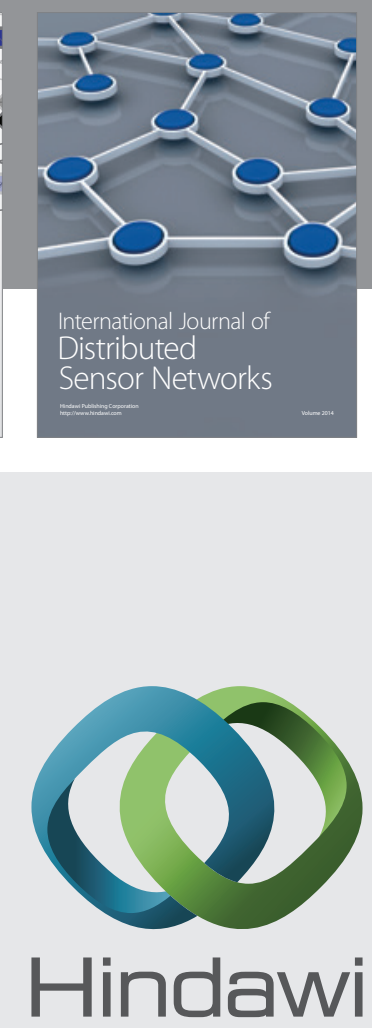

Submit your manuscripts at

http://www.hindawi.com
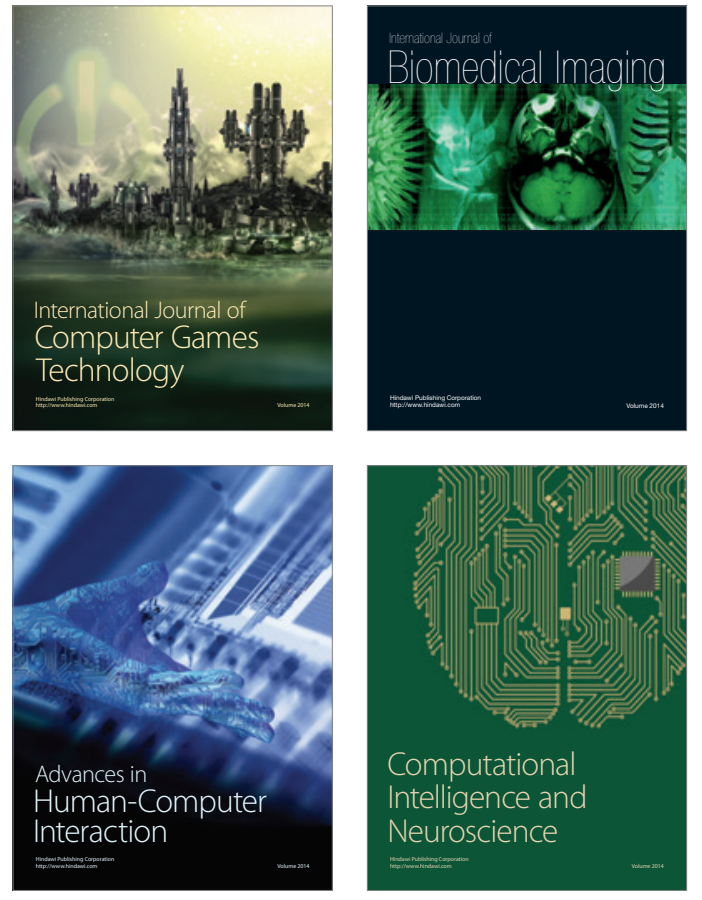
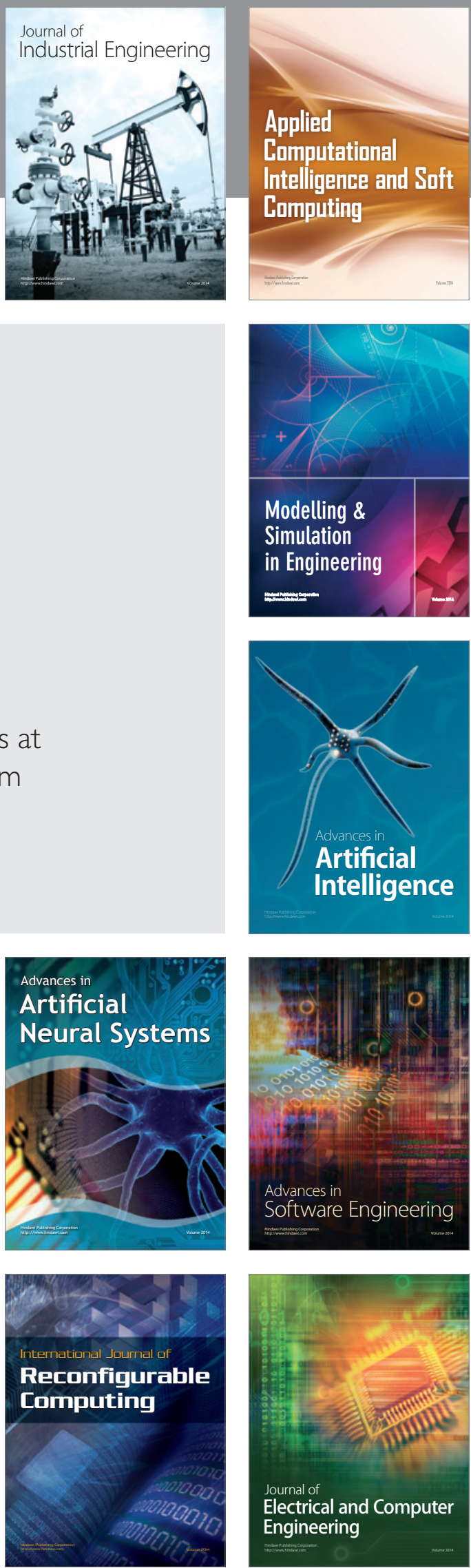